\title{
From the Human Spine to Hyperredundant Robots: The ERMIS Mechanism
}

\author{
Ioannis Georgilas $^{1}$ and Vassilios Tourassis ${ }^{2}$ \\ ${ }^{1}$ Bristol Robotics Laboratory, University of Bristol and University of the West of England, T Block, Frenchay Campus, \\ Bristol BS16 1QY, UK \\ ${ }^{2}$ Department of Robotics and Mechatronics, Nazarbayev University, 53 Kabanbay Batyr Avenue, Astana 010000, Kazakhstan
}

Correspondence should be addressed to Ioannis Georgilas; giannis.georgilas@brl.ac.uk

Received 30 April 2013; Accepted 9 July 2013

Academic Editors: L. Asplund, C.-C. Tsai, and Y. Zhou

Copyright (c) 2013 I. Georgilas and V. Tourassis. This is an open access article distributed under the Creative Commons Attribution License, which permits unrestricted use, distribution, and reproduction in any medium, provided the original work is properly cited.

\begin{abstract}
Mechatronics are occasionally inspired by nature for joint designs in order to exploit the advantages of the biological ones in terms of mobility and articulation. Within this context and based upon the human spine for structure and actuation, the authors will present a novel hyperredundant mechanism, named ERMIS. The muscle-skeletal system of the human trunk will be described and modelled, and the elements that are being replicated by the mechanical analog will be analysed. It will be shown that the vertebrae-intervertebral disk arrangement can be emulated by a spherical-type configuration, the proposed Disk-Ball-Disk joint. Furthermore, the muscle actuation system is being recreated by a system of wires and pulleys. The relevant kinematic models will be developed, and both simulation and experimental data to evaluate its operation will be demonstrated.
\end{abstract}

\section{Introduction}

Humans have always looked to nature for inspiration. In engineering and robotics research many mechanisms are imitations or reapplications of biological systems. This application in engineering design of natural processes and models is known as biomimicry or biomimetics. These mechanisms mimic the behaviour of biological organisms and offer many advantages over traditional designs.

There are, of course, practical limits to biomimicry. When looking to nature for instruction, one must remember that biological designs are built with unique materials, operate in distinct environments, and possess intricate cost functions to optimise their objectives. And nature is not always perfect. A better design is by necessity an evolutionary derivative of an existing design, and it is only measured against other designs that have already been built. Human invention is only limited by our own vision and can be optimised relative to all potential designs. Given these caveats, nature does remain an admirable teacher $[1,2]$.

Within this general context, a large number of biologically inspired concepts revolve around the issue of articulation and mechanical mobility. Most, if not all, mechanical joints used in engineering today are imitations or reapplications of biological ones. The simplest example is the revolute joint which is based on the structure of the joints of the upper and lower extremities of most vertebrate animals. Similar arguments can be made for the spherical and prismatic joints.

A remarkable paradigm of biologically inspired joint and articulation design example is the field of hyperredundant continuum robots $[3,4]$. Within this field of robotic research, novel ideas for continuum curvature robots [5] are being produced. The designs span from snake- and worm-based systems [6] to elephant trunks $[7,8]$, cephalopod tentacles [9], and animal tongues [10]. Similar systems are designed to be used in health applications as driving devices for catheters, canulas, and needles [11-13]. Also, many of these designs have found their way to industrial products [14-16].

The current work presents a novel design, based on a smart joint-actuation technique, for a continuous curvature manipulator, ERMIS. In previous work of the authors [17] a brief description of the characteristics of the mechanism and control architecture was given. In the current work detailed analysis of the similarities between the biomechanics 
of the human spine and the Disk-Ball-Disk (DBD) joint in the ERMIS system will be given along with the respective kinematic model. In Figure 1 the two systems are presented side by side.

The structure of the paper is as follows. In Section 2.1 a description of the structure and articulation of the human spine is given. In Section 2.2 the structural elements of the ERMIS mechanism are described, and the recreation of specific biological elements is noted. In Section 2.3 the kinematic models of both the human spine and ERMIS are presented and compared. Finally, in Section 3 simulation results of ERMIS work envelope and data from sample movements of ERMIS are presented, and its overall operation is evaluated.

\section{Materials and Methods}

2.1. The Human Spine. Human spine is a structure consisting of 24 vertebrae divided into 5 sections, the cervical, the thoracic, the lumbar, the sacral, and the coccygeal. This structure provides robust support for the entire body along with significant flexibility on flexion and extension. Two main groups of tissues can be identified in the human spine, the hard and soft ones.

The main hard tissue component of the spinal column is the vertebrae. It consists of an anterior block of bone, the vertebral body, and a posterior bony ring, known as the neural arch [18]. The first is the main part of the vertebrae, and the latter provides protection for the spinal cord and also support for some of the soft tissue components, Figure 2.

A variety of structures connecting vertebrae can be classified as soft tissue including facet joints, ligaments, intervertebral disks, and muscles [19]. The role of these soft tissue structures is to provide flexibility to the vertebral column while maintaining its integrity $[18,19]$. These tissues render the cervical spine compliant in that they allow for movement between the vertebrae. However, they are also responsible for limiting the range of many movements under normal conditions [18]. As a result, the range of motion of the spine varies from 5 to 45 degrees in the three rotation axes, the flexion/extension, the bending sideways, and the rotation around the human body axis.

The two soft tissues structures ERMIS is replicating are the intervertebral disks and the muscles actuating the spine. Intervertebral disk is a cartilaginous structure consisting of three parts, the nucleus pulposus in the centre, a mainly water based structure, the annulus fibrosus the outer part of the intervertebral disk which surrounds and protects the core, and the end plates which connect the disk with the vertebrae. Its function is to provide shock absorption for the trunk and also to act as the joint around which the vertebrae are performing their relevant motion. Because of pathogenic reasons the functionality of the intervertebral disks might be affected and the centre of the virtual spherical joint changes position [20].

Despite its incredible structure and functional abilities the spine is an unstable formation. Only under the coordinated forces generated by muscles the human trunk can maintain a particular posture and produce a specific movement. There are multiple muscle groups energizing the human spine and are grouped either by their proximity to the vertebrae as deep, intermediate, and superficial or by how many vertebrae they connect. The group that connects multiple vertebrae is called semispinalis spanning 4-6 vertebral joints, Figure 3. The latter group is the one responsible for the movement of the spine while the shorter ones are maintaining the spinal column's integrity.

2.2. ERMIS Mechanical Design. The ERMIS mechanism in analogy to the human spine is a serial linkage of special joints. Those joints are called Disk-Ball-Disk joints since their structural components are disks and steel spheres. In a manner similar to the human spine, each disk of these joints is actuated and the synchronised motion of the disks gives a uniform curvature to the manipulator. In the current section the description of the two components, joint and actuation system, will be given and the resemblance to the human spine will be underlined.

2.2.1. Disk-Ball-Disk Joint. Disk-Ball-Disk (DBD) joint mimics the combined structure of two subsequent vertebrae along with the intermediate intervertebral disk (spinal disk) structure. The role of the vertebrae is played by an aluminium disk with a curved surface on the upper and lower ends of it. The role of the spinal disk is assumed by a metallic steal ball which is encapsulated in the disks so as to allow the disk to flex, bend, and twist, without the fear of the ball falling out of the joint. The encapsulation of the ball is done by a proper opening on the top and bottom of the two curved surfaces of the disk, as shown in Figure 4.

The illustrated DBD joint, being a type of spherical joint, does possess a homogeneous, spherically shaped working envelope. Because of the underlying disk geometry, the hemispherical surface is reduced to a smaller but well-defined spherical section. This spherical section can be described by the angle $U$ which is the maximum angle the disks can revolve with respect to the main axis through the centre of the steel ball in Figure 4(b).

The angle $U$ can be calculated as a function (1) of the dimensions of the of the DBD joint, Figure 4(c):

$$
U=f\left(R, L, A, D, r, d_{h}, P e r\right),
$$

where $R$ is the curvature of the disk, $L$ is the thickness of the disk, $A$ is the finite width of flange, $D$ is the effective diameter of the disk, $r$ is the ball radius, $d_{h}$ is the diameter of disk foramen, and Per is the contact surface between disk and ball.

\subsubsection{Tendon-Driven Actuation. Actuation forces on the bio-} logical system are provided by the different muscles adjoint to the spinal column as described in Section 2.1. The equivalent in the mechanical arrangement is the use of tendons in the form of steel wires. This approach is being implemented as the wires can best emulate the properties of the muscles [21]; particularly, like the muscles, the wires can "pull" but cannot "push" and desired motion can be achieved by sets of them.

Remotely actuating continuum manipulators is a method that provides multiple benefits among which the size of the 


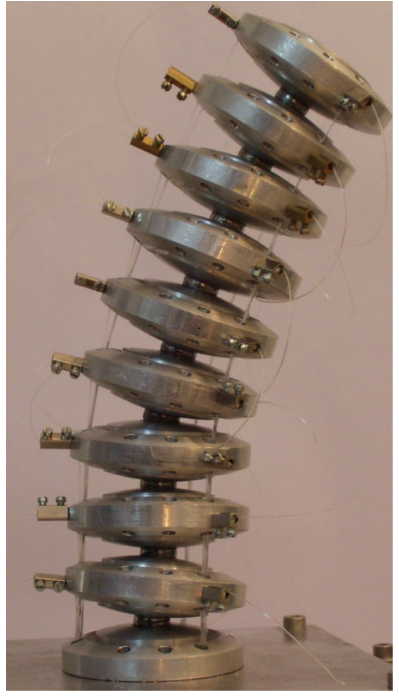

(a) ERMIS

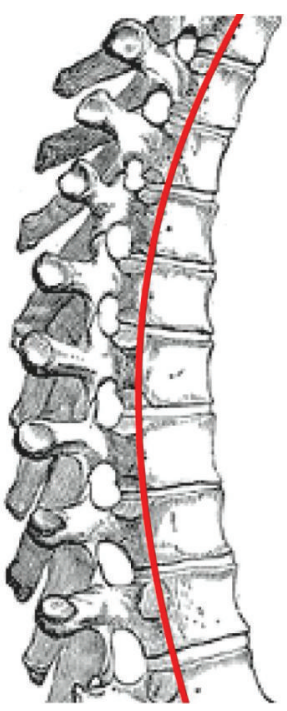

(b) Human Spine

FIGURE 1: Mechanical and biological systems. (a) ERMIS mechanism with 9 disks and (b) a similar human spine part with 9 vertebrae.

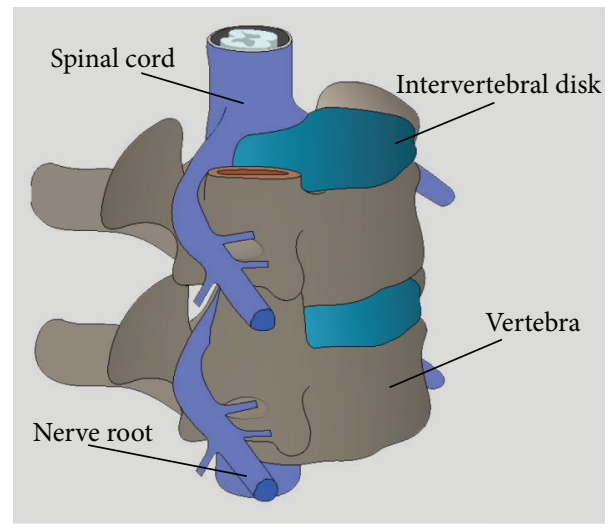

FIGURE 2: A link of the human spine. The relevant position of the vertebrae and the intervertebral disk can be seen along with special section of the vertebrae to allow the passage of the spinal cord.

manipulators body can remain small as in health applications [12]. The use of tendons also increase overall strength, since stronger and bulkier motors can be housed in the bigger base [22]. Within the same context, articulation can be increased with minimum increase of the overall manipulator body size since more DOFs can be added in the less size-restricted base [23].

Pulling forces are being applied to the wires using a pulley system. Since every disk of the ERMIS mechanism is actuated, a multilayered pulley is being implemented, Figure 5(a). The diameter of each level of the pulley is a function of the distance between the disks and the position of the disk in the DBD joints chain as follows:

$$
d_{n}=\frac{n \cdot l}{\pi}, \quad n=1 \cdots 9,
$$

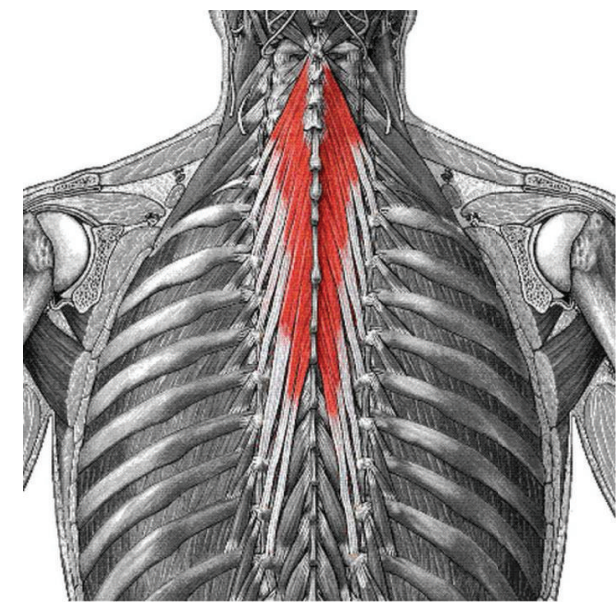

FIGURE 3: Muscles of the human spine. The semispinalis passing six or more vertebrae is being highlighted.

where $d_{n}$ is the diameter of the pulley layer $n$ and $l$ is the distance between two disks. $n$ in the case of the ERMIS prototype, as seen in Figure 1, is nine disks per section.

2.3. Kinematic Models. For the proper development of the kinematics of ERMIS an overview of the modelling of the human spine is needed. In the current Section the state of the art for modelling will be given and how the mechanical design of ERMIS grasps and emulates the biological system will be presented.

2.3.1. Modelling the Human Spine. In medical sciences the most prominent method to model the spinal column is by the fusion of 3D point cloud data from MRI and CAD designs. These two sets of information are being integrated to create 


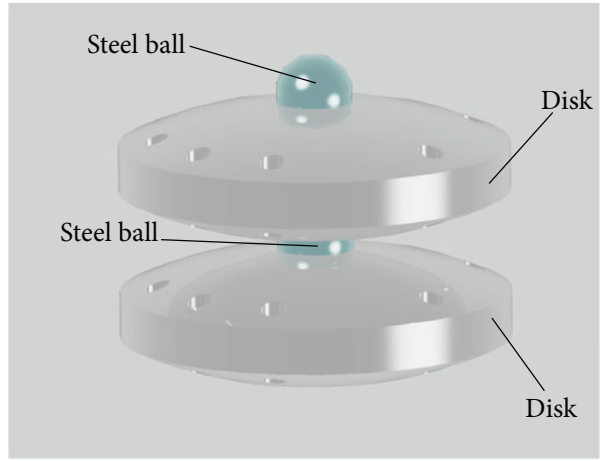

(a) Mechanical joints

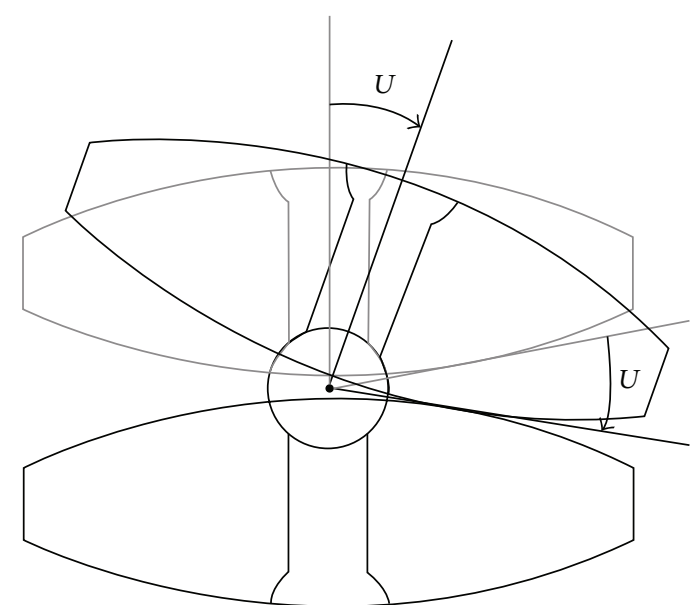

(b) Fundamental angle

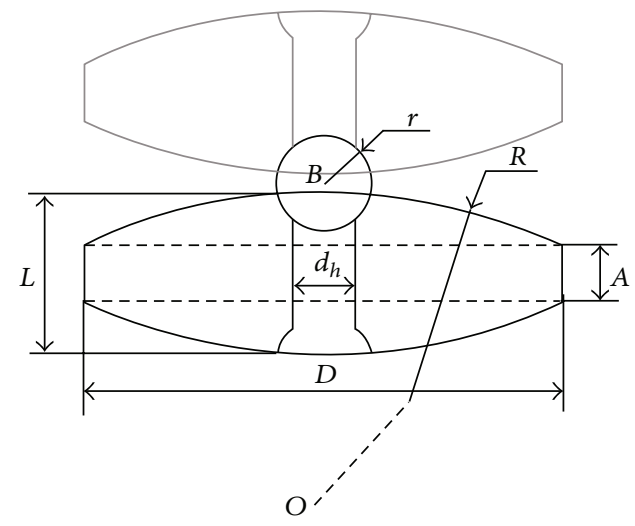

(c) Dimensions of the DBD joint

FIGURE 4: ERMIS joint, angle $U$, and the dimensions of the DBD joint that affect the range of motion.

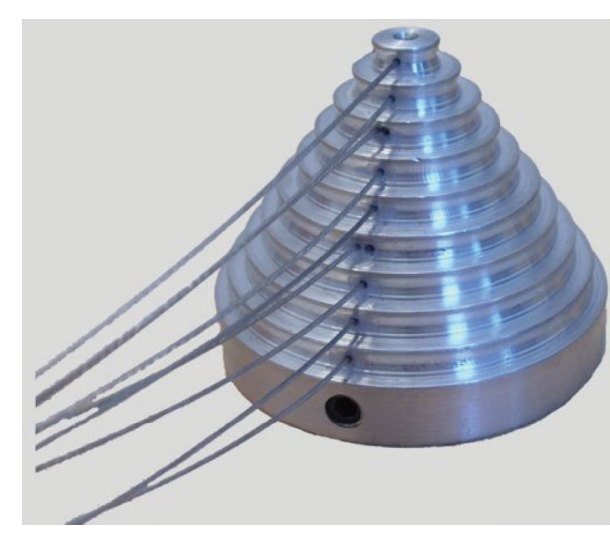

(a) Multilayered pulley

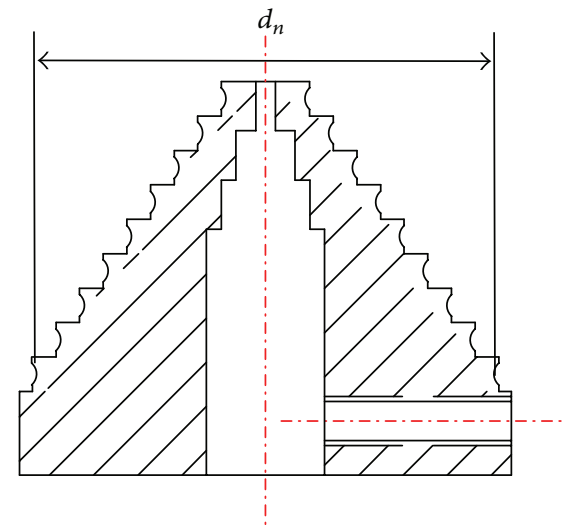

(b) Important dimensions

FIGURE 5: The multilayered pulley. (a) Picture with tendons attached and (b) design of it with the diameter of each layer given.

a precise model of the hard (vertebrae) parts of the human spine [24]. Often, data from the physiological analysis of soft tissue that informations is added to the generated model to enhance its accuracy [25]. This type of information can be further represented by the equivalent mechanical structures, specifically spring elements for ligaments and as Ahn suggested as a spherical joint in place of the intervertebral disk [20], Figure 6. 


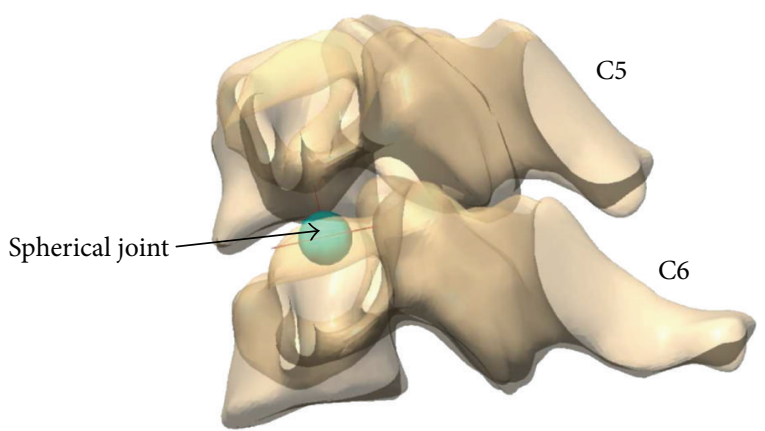

FIGURE 6: Spherical joint in the human spine. The location of the virtual spherical joint in a healthy sample.

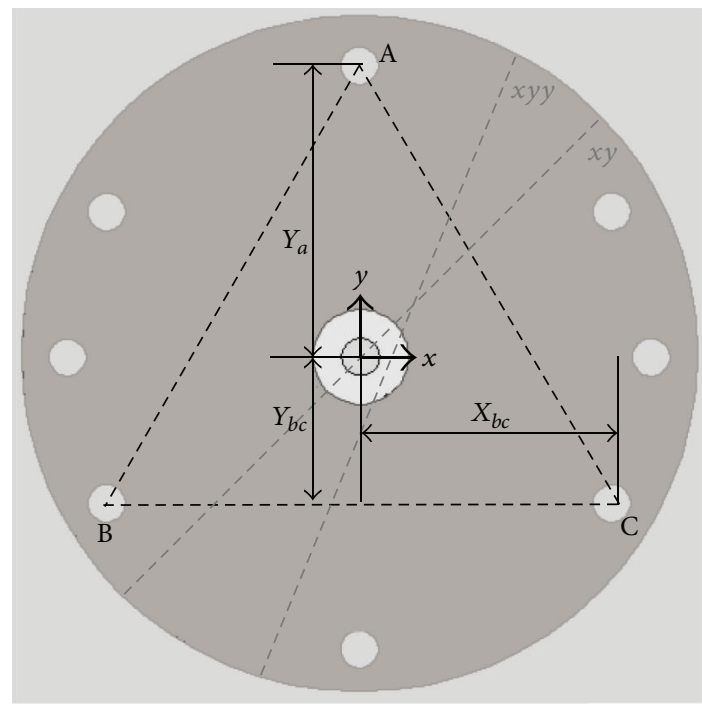

FIgURE 7: Actuation triangle. The openings through which the tendons pass along with dimensions and the coordinate system of the disk.

Given the detailed models and using finite element analysis (FEA), the behaviour of the human spine is predicted. For most of the cases in the medical literature, the simulation data is primarily focused on the impact from exercising excess forces to the human spine. The investigation of traumas inflicted on the human trunk helps designing the best possible methods for recovery and repair in case of spine accidents.

2.3.2. Impact of the Mechanical Design. The structure of ERMIS mechanical components significantly impacts the model describing the operation of the mechanism. The use of 3 tendons for force transfer creates some prerequirements in order for the manipulator to maintain stability. The movement of each disk must be symmetrical, and the tendons must operate in a synchronised manner. Because of the symmetrical nature of the movement, a system of agonists-antagonists between the tendons must be implemented. The possible sets of tendon movement, labeled according to Figure 7, can be seen in Table 1. Also, the ratio of displacement of each set

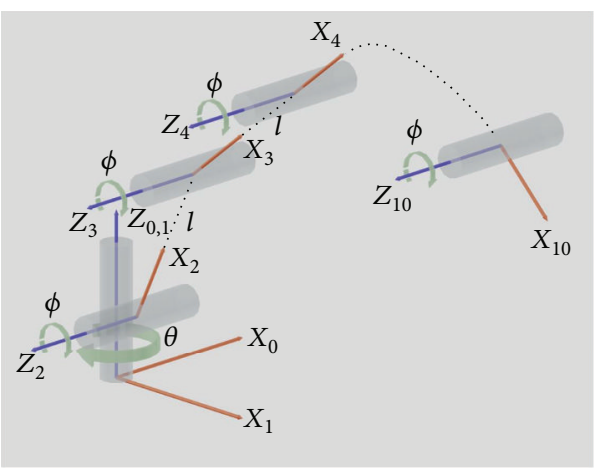

Figure 8: D-H coordinate systems of equivalent manipulator. The 10 coordinate systems for a 9 Disk-Ball-Disk joint section.

is given along with the respective axis of disk rotation. This symmetrical motion of the tendons simplifies the kinematics since for any given position and orientation of the end effector only two variables are needed, one for the agonist (tendon or set of tendons) and one for the antagonist (resp.).

None the less, 3 tendons are enough to achieve the desired articulation for a single section of ERMIS. More foramina exist in the disks to facilitate for tendons passing for next sections. As with the case of human spine, the rotation of the structure around its axis is limited but still facilitated by the existing tendons. Depending on the application, for example, spray painting [26], the rotation of the mechanism might not be necessary.

Furthermore, the thickness of of the disk as seen in (1) if adequate enough allows the tendons to refrain a straight position transforming ERMIS from a hyperredundant manipulator to a discrete mechanism. This equivalence simplifies kinematics analysis and allows the application of D-H formulation.

The concept of a virtual discrete manipulator to simplify calculations of end effector position and orientation has been proposed in [27]. However, the downside of this approach is that the virtual manipulator does not capture the real shape of the mechanism. Nevertheless, in ERMIS' case the serpentine structure of the mechanism and the actuation system, specifically the multilayered pulley, facilitate this transformation. The resulting discrete manipulator not only accurately maps end effector but also correlates the intermediate points of the joints to the overall body posture of ERMIS, Figure 8 .

2.3.3. ERMIS Kinematics. As referred to in the previous section, the independent variables for calculating the direct kinematics of ERMIS end effector are two: the displacement of tendons passing from openings $\mathrm{A}$ and $\mathrm{C}$ in Figure 7, $m_{1}$ and $m_{3}$, respectively, are being selected; the displacement of the third tendon, $m_{2}$ is coupled to $m_{3}$. Based on these two variables the final matrices for location and orientation of the end effector can be calculated as a series of computations:

$$
m_{1}, m_{3} \stackrel{\mathrm{RPY}}{\longrightarrow} \beta, \quad \gamma \stackrel{\text { Cartesian to }}{\stackrel{\text { Spherical }}{\longrightarrow}} \varphi, \quad \theta \stackrel{\mathrm{D}-\mathrm{H}}{\longrightarrow} P_{f}, R_{f} .
$$


TABLE 1: Agonist antagonist sets of tendons.

\begin{tabular}{lccc}
\hline Agonist(s) & Antagonist(s) & Ratio of displacement & Axis of disk rotation \\
\hline A & C and B & $1: Y_{b c} / Y_{a}$ & $x$ \\
B & A and C & $1: Y_{b c} / Y_{a}$ & $-x y y$ \\
C & A and B & $1: Y_{b c} / Y_{a}$ & $x y y$ \\
A & B & $1: 1$ & $-x y$ \\
B & C & $1: 1$ & $y$ \\
C & A & $1: 1$ & $x y$ \\
\hline
\end{tabular}

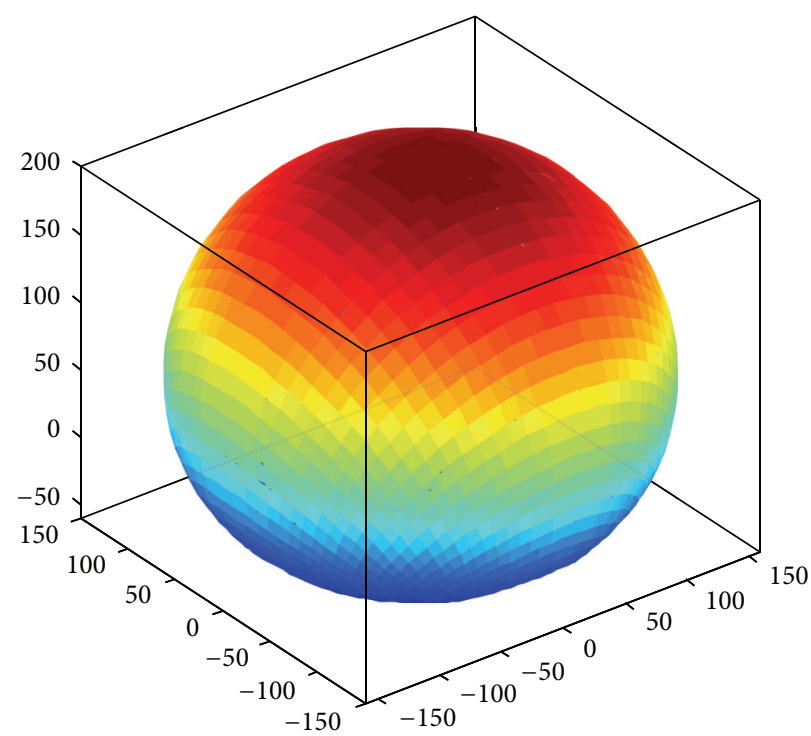

(a) Full work envelope

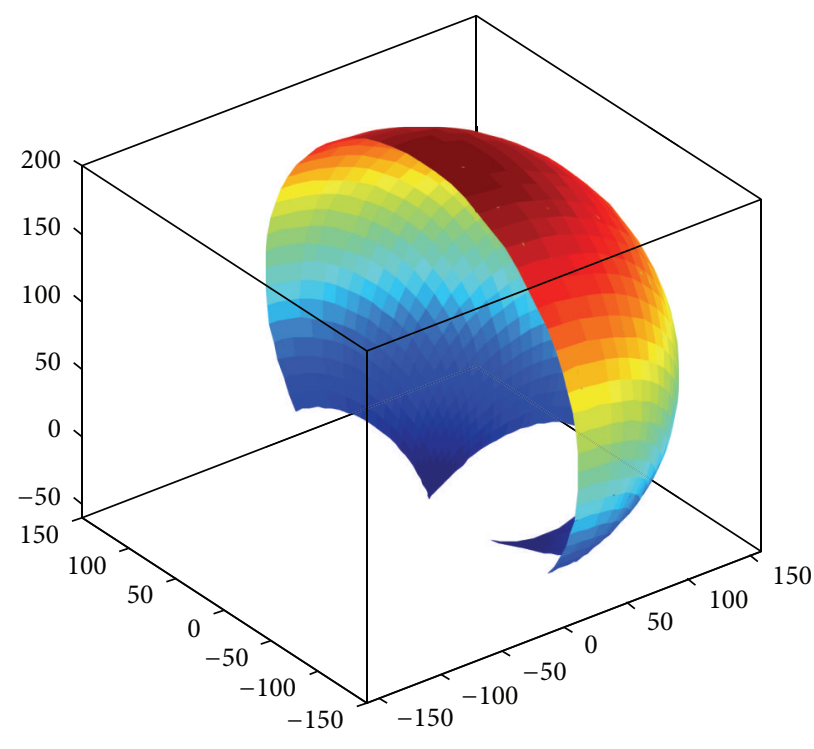

(b) Half work envelope

FIGURE 9: ERMIS work envelope. Both (a) the full envelope and (b) the half envelope can be seen. The latter allows for observing the inherent irregularities due to the actual joint limits.

TABLE 2: Equivalent rigid body manipulator D-H parameters.

\begin{tabular}{lcccc}
\hline$S_{i}$ & $\theta_{i}$ & $r_{i}$ & $d_{i}$ & $\alpha_{i}$ \\
\hline 0 & $\theta$ & 0 & 0 & 0 \\
1 & 0 & 0 & 0 & $-\pi / 2$ \\
2 & $\phi$ & 0 & $l$ & 0 \\
3 & $\phi$ & 0 & $l$ & 0 \\
$\vdots$ & $\vdots$ & $\vdots$ & $\vdots$ & $\vdots$ \\
10 & $\phi$ & 0 & $l$ & 0 \\
\hline
\end{tabular}

The first two steps are simple geometric formulations that, for reasons of brevity, are omitted in the current work. The resulting parameters $\phi$ and $\theta$ used for the DenavitHartenberg formulation, Table 2, can be seen in the following:

$$
\begin{aligned}
\varphi=- & \arctan \\
\times & \left(\left(\sigma_{1}-\sin \left(2 \arccos \left(\frac{Y_{a} m_{3}+Y_{b c} m_{1}}{2 Y_{a} X_{b c}}\right)\right)^{2}\right.\right. \\
& \left.\times\left(\sigma_{1}-1\right)\right)^{-1 / 2}
\end{aligned}
$$

$$
\begin{aligned}
& \times\left(\left(\frac{m_{1}{ }^{2}}{2 Y_{a}^{2}}-1\right)^{-1}\right. \\
& \left.\left.\times\left(\frac{\left(Y_{a} m_{3}+Y_{b c} m_{1}\right)^{2}}{2 Y_{a}^{2} X_{b c}^{2}}-1\right)\right)^{-1}\right), \\
& \theta=\arctan \left(\tan \left(2 \arcsin \left(\frac{m_{1}}{2 Y_{a}}\right)\right)\left(Y_{a} m_{3}+Y_{b c} m_{1}\right)\right. \\
& \times\left(\frac{4 Y_{a}^{2} X_{b c}{ }^{2}\left(\sigma_{2}-1\right)}{\left(Y_{a} m_{3}+Y_{b c} m_{1}\right)^{2}}-1\right) \\
& \left.\times\left(4 Y_{a} X_{b c} \sqrt{1-\sigma_{2}}\right)^{-1}\right),
\end{aligned}
$$

where $\sigma_{1}=\sin \left(2 \arcsin \left(m_{1} / 2 Y_{a}\right)\right)^{2}$ and $\sigma_{2}=\left(Y_{a} m_{3}+\right.$ $\left.Y_{b c} m_{1}\right)^{2} / 4 Y_{a}^{2} X_{b c}^{2}$.

Based on those two equivalent rotating joints the transformation matrix, of a single section with 9 DBD joints, can be developed as in (7). The components SS and SC exist 
as a result of the serial linkage of the equivalent rigid-link manipulator:

$$
\begin{gathered}
A_{0}=\left[\begin{array}{cccc}
\cos \theta & \sin \theta & 0 & 0 \\
-\sin \theta & \cos \theta & 0 & 0 \\
0 & 0 & 1 & 0 \\
0 & 0 & 0 & 1
\end{array}\right], \\
A_{1}=\left[\begin{array}{cccc}
1 & 0 & 0 & 0 \\
0 & 0 & 1 & 0 \\
0 & -1 & 0 & 0 \\
0 & 0 & 0 & 1
\end{array}\right], \\
A_{i}=\left[\begin{array}{cccc}
\cos \phi & \sin \phi & 0 & l \cos \phi \\
-\sin \phi & \cos \phi & 0 & l \sin \phi \\
0 & 0 & 1 & 0 \\
0 & 0 & 0 & 1
\end{array}\right],
\end{gathered}
$$

where $i=2 \cdots 10$.

Also

$$
\begin{aligned}
& T=\prod_{j=0}^{10} A_{j} \Longrightarrow T \\
& =\left[\begin{array}{cc|c}
R_{f} & P_{f} \\
\hline 000 & 0
\end{array}\right] \\
& =\left[\begin{array}{cccc}
\cos \theta \cos 9 \phi & \cos \theta \sin 9 \phi & -\sin \theta & l S S \cos \theta \\
\sin \theta \cos 9 \phi & \sin \theta \sin 9 \phi & \cos \theta & \text { lSS } \sin \theta \\
\sin 9 \phi & -\cos 9 \phi & 0 & \text { lSC } \\
0 & 0 & 0 & 1
\end{array}\right] \text {, }
\end{aligned}
$$

where

$$
\begin{aligned}
& S S=\sum_{i=1}^{9} \sin i \varphi=\frac{\sin (9 \varphi / 2) \sin 5 \varphi}{\sin (\varphi / 2)}, \\
& S C=\sum_{i=1}^{9} \cos i \varphi=\frac{\sin (9 \varphi / 2) \cos 5 \varphi}{\sin (\varphi / 2)} .
\end{aligned}
$$

Similarly the inverse kinematics can be computed by inverting (3). The resulting equations for the tendons displacements can be seen in the following:

$$
\begin{gathered}
m_{1}=2 Y_{a} \sin \left(\frac{\gamma}{2}\right), \\
m_{2}=-2\left(X_{b c} \sin \left(\frac{\beta}{2}\right)+Y_{b c} \sin \left(\frac{\gamma}{2}\right)\right), \\
m_{3}=2\left(X_{b c} \sin \left(\frac{\beta}{2}\right)-Y_{b c} \sin \left(\frac{\gamma}{2}\right)\right) .
\end{gathered}
$$

The coupling of displacements $m_{2}$ and $m_{3}$ is evident in (9b) and (9c). The coupling factor is the change in the sign of element $X_{b c} \sin (\beta / 2)$.

In a similar manner to the serial calculation of the position kinematics, the velocity kinematics of ERMIS can be computed. The flow of intermediate states can be seen in the following:

$$
\dot{m}_{1}, \dot{m}_{3} \stackrel{J_{\mathrm{RPY}}}{\longrightarrow} \dot{\beta}, \quad \dot{\gamma} \stackrel{J_{\mathrm{C} 2 S}}{\longrightarrow} \dot{\varphi}, \quad \dot{\theta} \stackrel{J_{\mathrm{D}-\mathrm{H}}}{\longrightarrow} \dot{x}, \dot{\omega} .
$$

The matrices $J_{\mathrm{RPY}}, J_{\mathrm{C} 2 S}$, and $J_{\mathrm{D}-\mathrm{H}}$ are given in (11), and $\dot{x}$ and $\dot{\omega}$ are the velocity vectors for linear and angular motions respectively:

$$
\begin{aligned}
& J_{\mathrm{RPY}}=\left[\begin{array}{cc}
\frac{Y_{b c}}{Y_{a} \cdot \zeta_{1}} & \frac{1}{\zeta_{1}} \\
\frac{1}{Y_{a} \sqrt{1-\left(m_{1}^{2} / 4 \cdot Y_{a}^{2}\right)}} & 0
\end{array}\right], \\
& J_{\mathrm{C} 2 S}=\left[\begin{array}{cc}
\frac{\cos \gamma \sin \beta}{\zeta_{2}} & \frac{\sin (\beta+\gamma)-\sin (\beta-\gamma)}{2 \cdot \zeta_{2}} \\
\frac{2 \cos \beta \tan \gamma}{\zeta_{3}}- & \frac{2 \sin \beta\left(\tan ^{2} \gamma+1\right)}{\zeta_{3}}
\end{array}\right] \text {, } \\
& J_{\mathrm{D}-\mathrm{H}}=\left[\begin{array}{ccc}
\overbrace{l S C \sin \theta} & l \cos \theta\left(S S-\sum_{i=0}^{k-2} i \sin \varphi\right) & 0 \\
l S S \cos \theta & -l \sin \theta\left(S C-\sum_{i=0}^{k-2} i \cos \varphi\right) & 0 \\
0 & -l\left(S C-\sum_{i=0}^{k-2} i \cos \varphi\right) & 0 \\
0 & \sin \theta & \sin \theta \\
0 & 0 & \cos \theta \\
1 & 0
\end{array}\right] \text {, }
\end{aligned}
$$

where $\zeta_{1}=X_{b c} \cdot \sqrt{1-\left(Y_{b c} \cdot m_{1}+Y_{a} \cdot m_{3}\right)^{2} / 4 \cdot X_{b c}^{2} \cdot Y_{a}^{2}}, \zeta_{2}=$ $\sqrt{\cos ^{2} \gamma \sin ^{2} \beta+\sin ^{2} \gamma}, \zeta_{3}=2 \tan ^{2} \gamma-\cos (2 \beta)+1$, and $S S$ and SC are from (8).

\section{Results and Discussion}

3.1. ERMIS Work Envelope. The equations for direct position kinematics have been tested under MATLAB to evaluate the work envelope of ERMIS. The resulting plot of all end effector points is given in Figure 9(a). This depiction was created by evaluating relationship (3). Angle $U$, the physical limitation of movement, was selected to be $20^{\circ}$, the actual manufactured value for ERMIS. Also, from the dimensions of the mechanism, $Y_{a}=25 \mathrm{~mm}, Y_{b c}=$ $12.5 \mathrm{~mm}$, and $X_{b c}=21.65 \mathrm{~mm}$. Given that ERMIS has 9 DBD joints, the parameters settings for $m_{1}$ and $m_{3}$ are [-100 $\mathrm{mm}, 100 \mathrm{~mm}]$ and $[-200 \mathrm{~mm}, 200 \mathrm{~mm}]$, respectively.

Figure 9(b) presents the internal structure of the work envelope. Special attention must be given to the irregular shapes at the end of the point plot. These structures are due to limiting cases in (7) specifically when the conditions in (12) are met:

$$
\lim _{m_{1} \rightarrow u_{m_{1}}, m_{3} \rightarrow u_{m_{3}}} \phi, \theta \quad \text { or } \quad \lim _{m_{1} \rightarrow l_{m_{1}}, m_{3} \rightarrow l_{m_{3}}} \phi, \theta,
$$

where $m_{1} \in\left[l_{m_{1}}, u_{m_{1}}\right]$ and $m_{3} \in\left[l_{m_{3}}, u_{m_{3}}\right]$.

3.2. Range of Motion. The range of motion of ERMIS manipulator has been evaluated with a set of sample movements. 

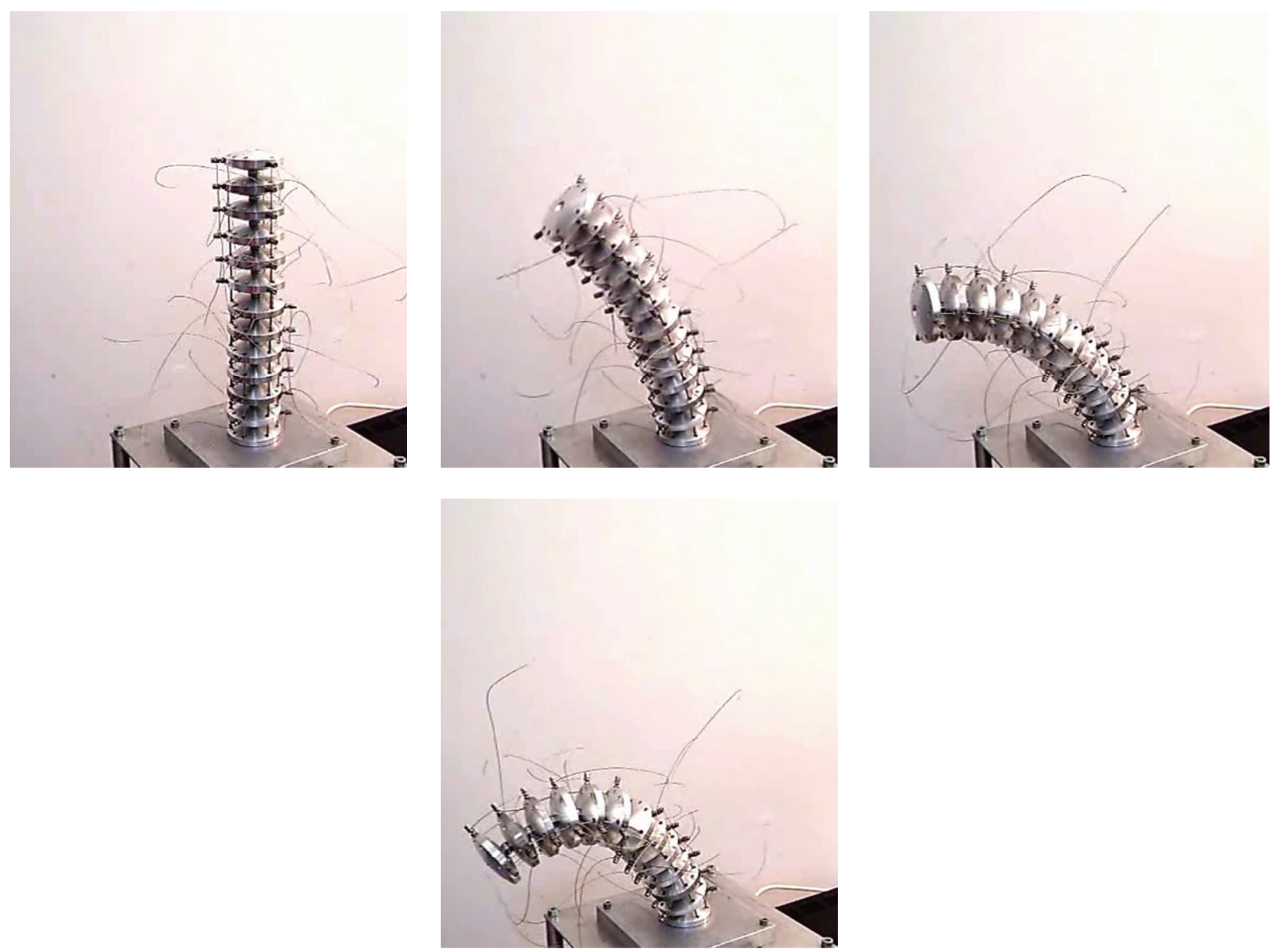

FIGURE 10: ERMIS prototype movement. The end-effector at $0^{\circ}$ (home position), $45^{\circ}, 90^{\circ}$, and $120^{\circ}$ (maximum bend) from the vertical.

Frames taken from these motions can be seen in Figure 10. In those pictures the mechanism is moving from the home position $\left(0^{\circ}\right)$ to three consecutive points where the coordinate system of the end effector is being rotated around the base's system by $45^{\circ}, 90^{\circ}$, and $120^{\circ}$.

\section{Conclusions}

With the current work, a novel mechanism based in the human spine, ERMIS, has been presented. The key features of the biological system have been analysed, and the equivalent mechanical interpretations have been described.

Furthermore, based on the medical literature the modelling of human trunk has been given and the assumption of the intervertebral disk as a spherical joint is being presented. Based on this hypothesis, the operation of the DBD joint has been evaluated and a kinematically accurate model of ERMIS is being developed.

The experimental data from both the simulation of ERMIS motion and the movie life frames collected from actual motion of the mechanism shows that the motion of ERMIS follows the model developed.
Future work involves a detailed analysis of the singularity points in the work envelope of ERMIS and the development of a motion controller to allow implementation of ERMIS in real-life applications.

\section{References}

[1] J. F. V. Vincent and D. L. Mann, "Systematic technology transfer from biology to engineering," Philosophical Transactions of the Royal Society A, vol. 360, no. 1791, pp. 159-173, 2002.

[2] J. O. Wilson and D. Rosen, "Systematic reverse engineering of biological systems," in Proceedings of the 19th International Conference in Design Theory and Methodology and 1st International Conference in Micro and Nano Systems, ASME International Design Engineering Technical Conferences and Computers and Information in Engineering Conference (IDETC/CIE '07), vol. 3, part A, pp. 69-78, Las Vegas, Nev, USA, September 2007.

[3] G. Robinson and J. B. C. Davies, "Continuum robots-a state of the art," in Proceedings of the IEEE International Conference on Robotics and Automation (ICRA '99), pp. 2849-2854, May 1999.

[4] I. D. Walker, "Continuous backbone "continuum" robot manipulators," ISRN Robotics, vol. 2013, Article ID 726506, 19 pages, 2013. 
[5] R. J. Webster III and B. A. Jones, "Design and kinematic modeling of constant curvature continuum robots: a review," International Journal of Robotics Research, vol. 29, no. 13, pp. 1661-1683, 2010.

[6] S. Hirose, Biologically Inspired Robots (Snake-Like Locomotor and Manipulator), Oxford University Press, London, UK, 1993.

[7] R. Cieślak and A. Morecki, "Elephant trunk type elastic manipulator-a tool for bulk and liquid materials transportation," Robotica, vol. 17, no. 1, pp. 11-16, 1999.

[8] M. W. Hannan and I. D. Walker, "The "elephant trunk" manipulator, design and implementation," in Proceedings of the IEEE/ASME International Conference on Advanced Intelligent Mechatronics (AIM '01), vol. 1, pp. 14-19, July 2001.

[9] I. A. Gravagne, C. D. Rahn, and I. D. Walker, "Large deflection dynamics and control for planar continuum robots," IEEE/ASME Transactions on Mechatronics, vol. 8, no. 2, pp. 299307, 2003.

[10] H. Takanobu, T. Tandai, and H. Miura, "Multi-DOF flexible robot base on tongue," in Proceedings of the IEEE International Conference on Robotics and Automation (ICRA '04), pp. 26732678, May 2004.

[11] A. M. Andruska and K. S. Peterson, "Control of a snakelike robot in an elastically deformable channel," IEEE/ASME Transactions on Mechatronics, vol. 13, no. 2, pp. 219-227, 2008.

[12] D. B. Camarillo, C. F. Milne, C. R. Carlson, M. R. Zinn, and J. K. Salisbury, "Mechanics modeling of tendon-driven continuum manipulators," IEEE Transactions on Robotics, vol. 24, no. 6, pp. 1262-1273, 2008.

[13] D. C. Rucker, R. J. Webster III, G. S. Chirikjian, and N. J. Cowan, "Equilibrium conformations of concentric-tube continuum robots," International Journal of Robotics Research, vol. 29, no. 10, pp. 1263-1280, 2010.

[14] T. J. Drozda, "The spine robot... the verdict's yet to come," Manufacturing Engineering, vol. 93, no. 3, pp. 110-112, 1984.

[15] G. Immega and K. Antonelli, "The KSI tentacle manipulator," in Proceedings of the IEEE International Conference on Robotics and Automation, pp. 3149-3154, May 1995.

[16] R. Buckingham and A. Graham, "Snaking around in a nuclear jungle," Industrial Robot, vol. 32, no. 2, pp. 120-127, 2005.

[17] I. P. Georgilas and V. D. Tourassis, "ERMIS-a novel biologically inspired flexible robotic mechanism for industrial applications," in Proceedings of the IEEE/ASME International Conference on Advanced Intelligent Mechatronics (AIM '09), pp. 1504-1509, Singapore, July 2009.

[18] A. A. White and M. M. Panjabi, Clinical Biomechanics of the Spine, Lippincott Williams \& Wilkins, Philadelphia, Pa, USA, 1990.

[19] N. Yoganandan, S. Kumaresan, and F. A. Pintar, "Biomechanics of the cervical spine. Part 2. Cervical spine soft tissue responses and biomechanical modeling," Clinical Biomechanics, vol. 16, no. 1, pp. 1-27, 2001.

[20] H. Ahn, A virtual model of the human cervical spine for physicsbased simulation and applications [Ph.D. thesis], The University of Tennessee Health Science Center, 2005.

[21] S. K. Mustafa, G. Yang, S. H. Yeo, W. Lin, and I. M. Chen, "Selfcalibration of a biologically inspired 7 DOF cable-driven robotic arm," IEEE/ASME Transactions on Mechatronics, vol. 13, no. 1, pp. 66-75, 2008.

[22] K. Ning and F. Worgotter, "Control system development for a novel wire-driven hyper-redundant chain robot, 3D-trunk," IEEE/ASME Transactions on Mechatronics, vol. 17, no. 5, pp. 949-959, 2012.
[23] O. Robotics, "Snake-arm robots access the inaccessible," Nuclear Technology International, vol. 1, pp. 92-94, 2008.

[24] G. Monheit and N. I. Badler, "A kinematic model of the human spine and torso," IEEE Computer Graphics and Applications, vol. 11, no. 2, pp. 29-38, 1991.

[25] C. U. de Jongh, A. H. Basson, and C. Scheffer, "Dynamic simulation of cervical spine following single-level cervical disc replacement," in Proceedings of the Annual International Conference of the IEEE Engineering in Medicine and Biology, pp. 4289-4292, August 2007.

[26] I. P. Georgilas and V. D. Tourassis, "Quality issues in enameling of ceramic industry products," in Proceedings of the IEEE International Conference on Industrial Engineering and Engineering Management (IEEM '07), pp. 1225-1230, IEEE, Singapore, December 2007.

[27] B. A. Jones and I. D. Walker, "Kinematics for multisection continuum robots," IEEE Transactions on Robotics, vol. 22, no. 1, pp. 43-55, 2006. 

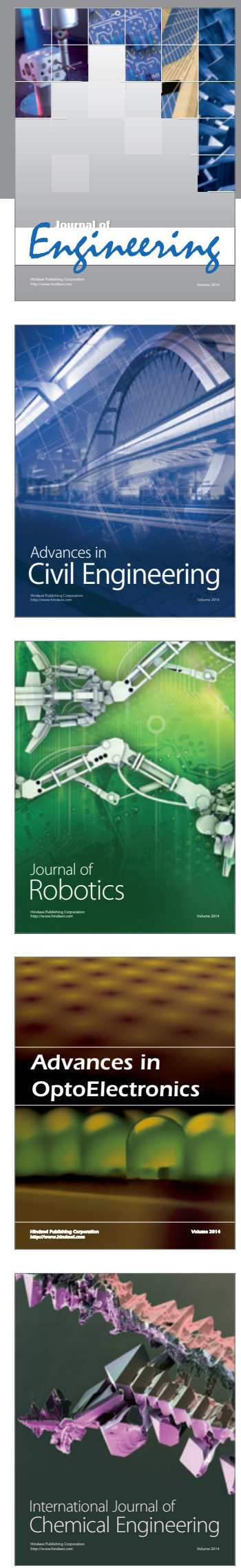

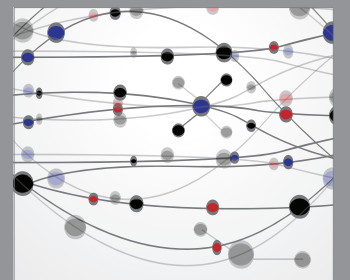

The Scientific World Journal
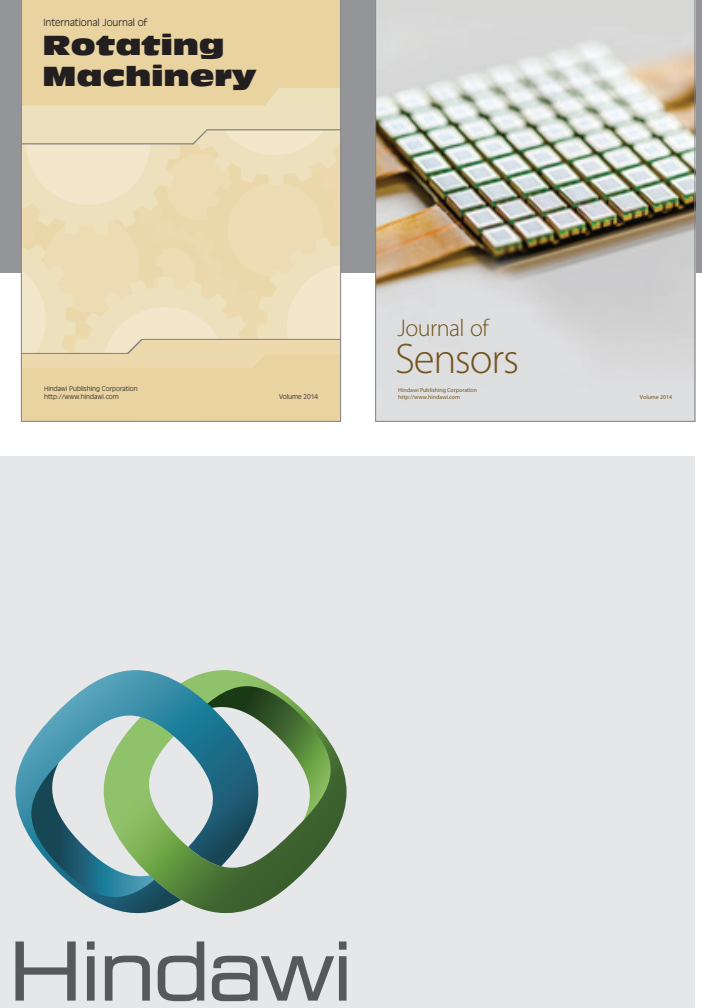

Submit your manuscripts at http://www.hindawi.com
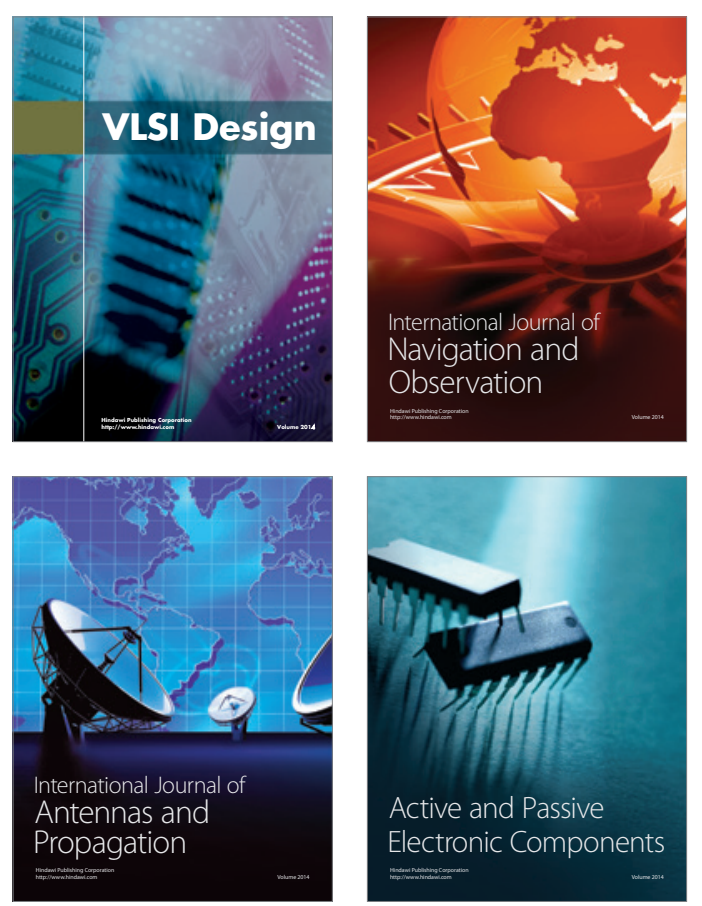
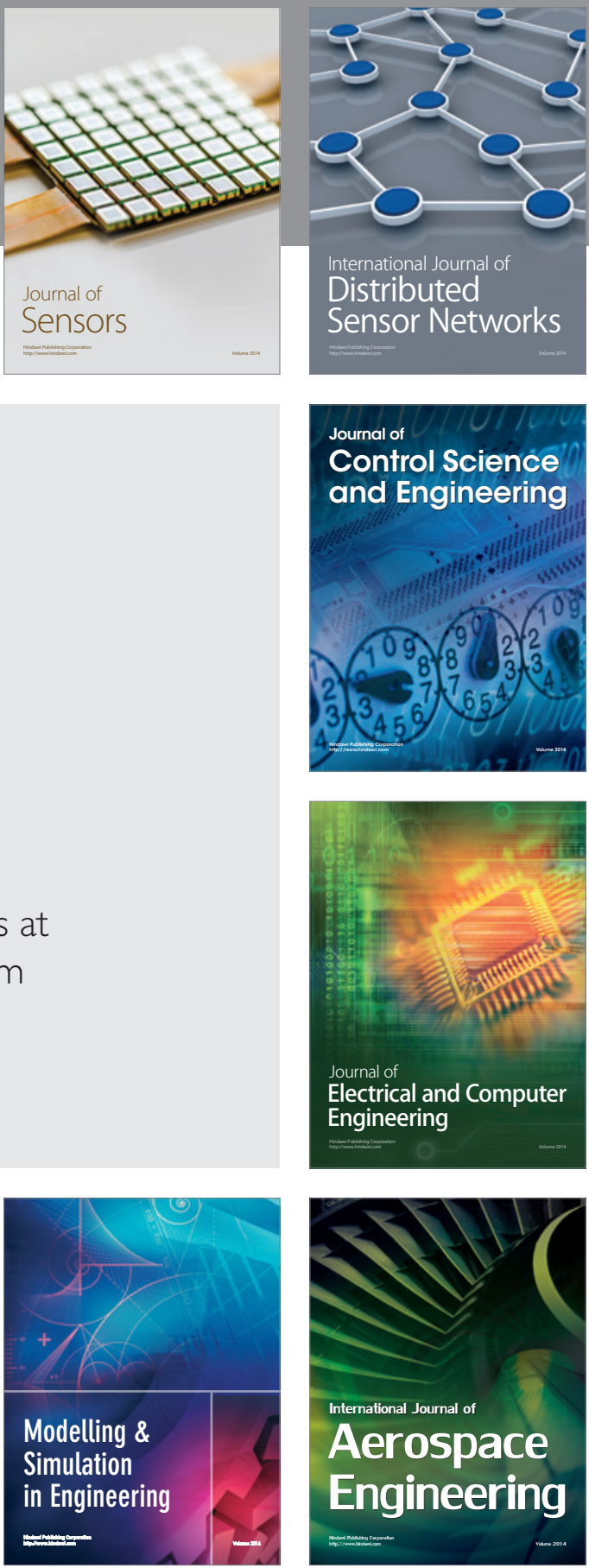

Journal of

Control Science

and Engineering
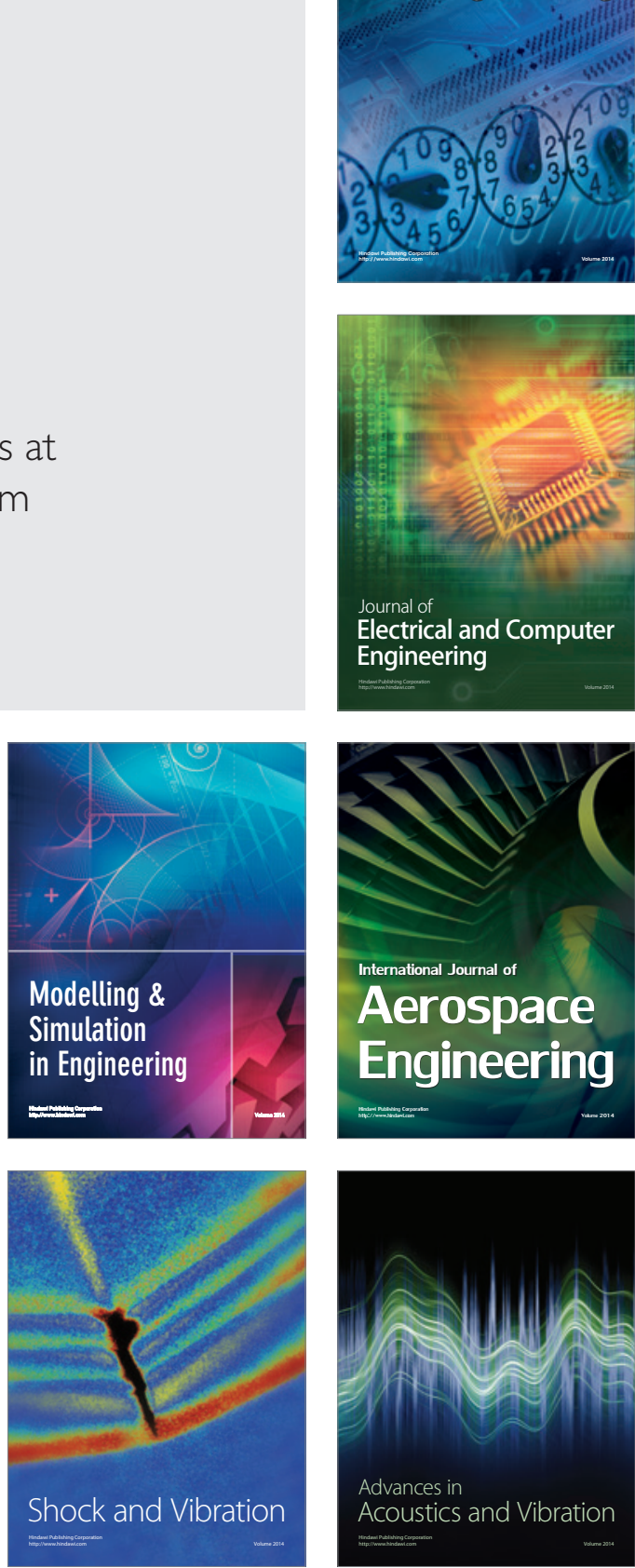